Kragujevac Journal of Mathematics

Volume 38(1) (2014), Pages 5-21.

\title{
A CONCISE MINI HISTORY OF GEOMETRY
}

\author{
LEOPOLD VERSTRAELEN
}

\section{Origin And development in Old Greece}

Mathematics was the crowning and lasting achievement of the ancient Greek culture. To more or less extent, arithmetical and geometrical problems had been explored already before, in several previous civilisations at various parts of the world, within a kind of practical mathematical scientific context. The knowledge which in particular as such first had been acquired in Mesopotamia and later on in Egypt, and the philosophical reflections on its meaning and its nature by "the Old Greeks", resulted in the sublime creation of mathematics as a characteristically abstract and deductive science. The name for this science, "mathematics", stems from the Greek language, and basically means "knowledge and understanding", and became of use in most other languages as well; realising however that, as a matter of fact, it is really an art to reach new knowledge and better understanding, the Dutch term for mathematics, "wiskunde", in translation: "the art to achieve wisdom", might be even more appropriate.

For specimens of the human kind, "nature" essentially stands for their organised thoughts about sensations and perceptions of "their worlds outside and inside" and "doing mathematics" basically stands for their thoughtful living in "the universe" of their idealisations and abstractions of these sensations and perceptions. Or, as Stewart stated in the revised book "What is Mathematics?" of Courant and Robbins: "Mathematics links the abstract world of mental concepts to the real world of physical things without being located completely in either". Amongst the main Greek schools and their mathematical heroes, one has: Milete (now Turkey) with Thales $(\sim-624$ / -548), Croton (now Italy) with Pythagoras ( -566/-497), Athens with Eudoxos $(\sim$

Key words and phrases. Geometry, Curvature, Symmetry.

2010 Mathematics Subject Classification. Primary: 01A05. Secondary: 53B25, 53C40.

Received: April 10, 2014.

The author thanks the Center for Scientific Research of the Serbian Academy of Sciences and Arts and the University of Kragujevac for their partial support of his research done for this paper. 
-408/-355), Alexandria (now Egypt) with Euclides ( -365/-285) and Apollonius $(\sim$ -260/-170), and Syracuse (now Sicilia) with Archimedes ( -287/-212).

From the introduction of the book by Courant and Robbins comes the quote: "Mathematics as an expression of the human mind reflects the active will, the contemplative reason, and the desire for aesthetic perfection. Its basic elements are logic and intuition, analysis and construction, generality and individuality. Though different traditions may emphasize different aspects, it is only the interplay of these antithetic forces and the struggle for their synthesis that constitute the life, usefulness, and supreme value of mathematical science". Thales is considered as one of the founding fathers of mathematics and his theorem essentially concerns the basic notion of similarity. The theorem of Pythagoras, [in any rectangular triangle with sides of lengths $A$ (for the hypothenusa) and $B, C$ (for the sides along the right angle): $A^{2}=B^{2}+C^{2}$, remains the cornerstone of the geometry of the human kind. In the school of Pythagoras, it was shown that the side $(-)$ and the diagonal $(-)$ of a regular 5-gon, which was in the logo of this school, are incommensurable line segments, i.e. that their ratio (-"over" -) is an irrational number, ["a decimal number running on to infinity after the decimal sign, comma or point, without thereby, starting at some place before or after this sign, for always to repeat a fixed sequence of ciphers of any length"], namely the golden section $\varphi=0,618 \ldots$, which turned out to be of ever actual distinguished importance in geometry, in nature and in the arts alike. The theory of the geometrical continuum of Eudoxos properly dealt with the incorporation in the mathematical reality of such pairs of line segments -which earlier had been tacitly assumed not to exist-, thereby setting the deductive-postulational trend which aims for "security" of the mathematical reasoning as necessary counterpart for otherwise mostly intuitive, and eventually too loose ways of imagining and exploring problems in mathematics and in its applications. The crystallisation of this mode of thinking in Euclid's "Elements", of which the purpose was to survey the mathematics of its time, became a landmark in the history of our civilisation. And Euclid's complementing "Optics" may be seen as one of the first systematic studies of human vision. The parallel postulate in planar Euclidean geometry inevitably was pretty intriguing for many a scholar, already since Euclid's time, because "it involves a happening taking place at infinity, and our kind is not able to see so far". And this was so good, because, as formulated by Coolidge in his book "A History of Geometrical Methods": "It is to the doubts about Euclid's parallel postulate and the efforts of many thinkers to settle these doubts that we owe the whole modern abstract conception of mathematical science". Both from the angle of perspective and from the use of sundials, the conic sections came in focus and Apollonius' studies of them became the geometrical classics which played a vital role in the discovery of Kepler's laws of the planetary motions. Archimedes studied geometry and the laws of nature by making use of the powerful reasoning involving "infinitesimals", thereby anticipating the differential and integral calculus. 


\section{EUROPEAN RENAISSANCE AND GLOBAL MODERN DEVELOPMENT}

What remained preserved of the mathematics and of the exact, medical and human sciences of the ancient times, in original form and in translations, in Byzantium, in European monasteries and elsewhere, and also the extensions of and additions to this heritage in general by the Chinese, Japanese, Jewish and other civilisations, and, in mathematics, in particular by the Hindu and the Arabic contributions on trigonometry and on algebra, after a long dark period, again became sources of reflection and inspiration for European scholars like, amongst others, Fibonacci, Oresme, da Vinci and Dürer. And one of the historical lines of significant new developments in mathematics and its applications traces back to Gemma Frisius (1508-1555), a professor at provincial Leuven which is situated a little distance south of Antwerpen, the then cultural and mercantile center of the Low Counties and "abroad". Frisius studied the triangulations of surfaces, (related to elementary cartography), and explicitly wrote down some of the first tables of interests (to be paid on loans according to a new catholic deviation from the former Christian loaning systems), and so, for compound interests, basically tables of exponential functions. Together with his student Mercator (1512-1594), he studied the stereographic projection and made celestial and worldly globes. And later Mercator composed his first collection of charts of parts of the earth and introduced for it the name "atlas". Around 1550 he started to work at his map of the world, specially designed to serve navigation, hereby using what became known as "Mercator's projection", i.e. the conformal map that turns loxodromes on a sphere into straight lines on their planar image, and which analytically involves logarithmic functions. And in this environment Simon Stevin (1548-1620) learned and matured to become one of the great geometers in the period between Archimedes and Newton. One of his earliest booklets was printed by Plantijn at Antwerp and, as far as known, was the first real publication of tables of interests, (and he wrote it to inform the normal people of his time about the actual meaning of such interests in order to prevent them from being lured into some unpleasant traps). His booklet "De Thiende" was printed by Plantijn at Leiden in 1585 and definitely settled the concrete performance of all calculations involving real numbers in terms of elementary computations exclusively involving natural numbers, by making use of the decimal system, which system he moreover strongly advocated to be used universally for the practical benefit of international commerce. Among Simon Stevin's manifold other achievements in the sciences, one may remember in particular his composition law of forces, "the parallelogram rule", i.e. essentially the equilibrium illustrated in his logo with as motto "Wonder en is gheen wonder", and which is at the basis both of abstract algebra and of multidimensional geometry. Optics and the theory of vision, amongst others the study of perspective, were further developed too, trigonometry also hereby showing its use e.g. in Snellius' law of refraction. In physics, the Copernican cosmology was geometrically explicited in the observed laws of Kepler, which would later find their mathematical description in Newton's mechanics, of which the 
central idea is to geometrically conceive the forces which act on moving bodies as the curvature vector fields of their trajectories in space, up to a multiplicative constant which corresponds to the masses of these bodies.

Archimedes' classical problem to determine the tangent lines to arbitrary planar curves at any of their points and Simon Stevin's problem concretely to find all the spherical loxodromes were at the origin of the creations of the differential and of the integral calculus, i.e. of the mathematical discipline called analysis. Essentially this became possible only by the systematic use of the co-ordinate method of Descartes' "Géométrie" of 1637, which itself likely resulted from the human perceptual difficulties with the notion of parallelism. Hereby the delicate role played by the axiomatic foundation of synthetic geometry basically was taken over by the geometrical foundation of the real number system and, paraphrasing Descartes, by the fact that all Euclidean geometrical problems can be reduced to the knowledge of the distance between any two points (the theorem of Pythagoras). And, for most practical purposes, the procedures of analysis were reduced to the so-called theorem of Taylor-Maclaurin to which Newton, at Cambridge, was inspired by the consideration of the real functions in analogy to the consideration of the real numbers in the decimal system. Already before, his teacher Barrow had obtained the fundamental theorem of analysis which interrelates integration and differentiation as mutually inverse operations. Around 1670, Newton moreover could analytically determine the curvature of any Euclidean planar curve at any of its points, hereby following the considerations of amongst others Descartes and Kepler on the osculating approximation of general curves by circles and of Huygens on caustics etc. related to his studies of pendulum clocks.

And, since 1760, with the works of Euler, Monge, Meusnier, Dupin, Rodrigues, Germain, Casorati, etc., accordingly the curvature behaviour of surfaces in Euclidean spaces geometrically and analytically became more and more clear, and in this context Gauss discovered the $2 D$ classical non-Euclidean geometries, the hyperbolic versions of which having been treated also axiomatically and independently by Lobachevsky and by Bolyai. According to Galileo, "The book of Nature is written in a mathematical language and the characters are triangles, circles and other geometrical objects", i.e. many natural phenomena can be described in terms of geometrical notions and, in particular, in terms of curvatures, of which in the mean time the analytical expressions were known. Hence, since moreover many of these phenomena concern realisations of various kinds of extremal values and of certain equilibria or symmetries within given situations, the solutions of many variational principles of Maupertuis, Fermat, Leibniz, Bernoulli's, etc., often by applying the variational calculus of Euler and Lagrange, yielded spectacular developments in natural philosophy. For instance, several cycloids, catenaries and spirals, with most in particular the spira mirabilis (the natural logarithmic spiral), and, for instance, surfaces of constant mean curvature and surfaces of constant Gauss curvature and their geodesics and eventual loxodromes, showed up in this way as curves and surfaces of very special importance indeed, for geometry and for the applications of geometry in science and in technology alike. 
Gauss' 1827 Göttingen "Disquisitiones generalis circa superficies curvas" contained the germ of the notion of differential manifolds, i.e. of the mathematical field of "global analysis", and it made the crucial distinction for surfaces in Euclidean $3 D$ spaces between their intrinsic and their extrinsic geometrical properties, i.e. between the properties which exclusively depend on the distances between the points of these surfaces such as these distances result from the measurements of the lengths of the curves which lie completely on these surfaces and which connect these points and the properties which basically depend on the shapes that these surfaces assume in the ambient Euclidean spaces, or still on "the looks" of these surfaces when observing them in space. As described by Dombrowski in his historical comments on Gauss' geometrical disquisitiones, Gauss delayed the presentation of this fundamental work till he had obtained an analytical proof of the theorema egregium -the invariance of his "Krümmung" $K$ under isometrical surface transformations- in terms of general curvilinear surface co-ordinates, while in the course of his earlier investigations on general conformal transformations of surfaces, (his extension of the stereographic and Mercator projections of spheres on planes, whereby stating that "interesting maps between two arbitrary surfaces would indeed better be similar in their infinitesimal parts"), he already had found such proof in terms of isothermal co-ordinates; and, by the way, these investigations were at the basis of complex analysis. Roughly speaking, "global analysis" deals with the essentials of the traditional fields of algebra and analysis. And the awareness of an intrinsic geometry of surfaces in $3 D$ Euclidean space amounts to the awareness of the existence of "abstract" RiemannFinsler geometries on the not too weird differential manifolds. The establishment of these geometries was done independently by Riemann and, respectively, Helmholtz, in their "Ueber die Hypothesen, respectively, Tatsachen, welche der Geometrie zu Grunde liegen", published in 1866 (though Riemann's lecture at Göttingen with this title was delivered already in 1854), respectively, in 1868, who were hereby reflecting in the spheres of natural philosophy, and, in particular, about the physical spacetime, respectively, about human visual perception. And, in his "Ueber die tatsächlichen Grundlagen der Geometrie (1866-1869)", Helmholtz drew attention to the approach first taken by Gauss with the purpose to finally settle the Euclidean parallel postulate problem, not in the least done so in order to solidly attain some "objective control of the geometry of our kind's most basic visual and motoric senses", and later followed by Riemann in his generalisation of the intrinsic geometry of the $2 D$ surfaces $M^{2}$ in Euclidean $3 D$ spaces $E^{3}$ to the $n D$ abstract Riemannian geometry. That is, to begin with, in a way to carry over Descartes" "programme" which had built up the whole of the classical $2 D$ Euclidean geometry solely starting from the determination of the distances between pairs of points by means of the theorem of Pythagoras expressed in a $2 D$ Cartesian co-ordinate system, (and which programme is completely the same for all dimensions), to the inner geometry of surfaces $M^{2}$ in $E^{3}$, whereby the surfaces are described by curvilinear, say $(u, v)$ co-ordinates or charts and whereby 
"a geometrical structure" is defined on these surfaces $M^{2}$ by their first or metrical fundamental form $g$ or originally called line element $d s$ as being expressed by the infinitesimal distance function which is naturally induced on these surfaces $M^{2}$ from the standard theorem of Pythagoras' Euclidean distance function of the ambient space $E^{3}$, i.e. via a generalised theorem of Pythagoras on $M^{2}$ : a squared infinitesimal line element given by a general homogeneous quadratic polynomial in infinitesimal changes $d u, d v$ of the curvilinear co-ordinates, $d s^{2}=E d u^{2}+2 F d u d v+G d v^{2}$. Then, locally, the $2 D$ Euclidean geometry and the classical elliptical $2 D$ non-Euclidean geometry and the classical hyperbolic $2 D$ non-Euclidean geometry of LobachevskyBolyai are realised on surfaces $M^{2}$ in $E^{3}$ with constant Gauss curvature $K=\mathrm{o}$ (i.e. on "developpable surfaces"), $K>$ o (like on spheres) and $K<$ o (like on pseudospheres or tractroids), respectively. And, where for the Riemann-Finsler $n D$ geometries both Riemann and Helmholtz started from analytically $n$-fold extended "Mannigfaltigkeiten" for their basic spaces, i.e. both used systems of local co-ordinates $\left(x^{1}, x^{2}, \ldots, x^{n}\right)$-essentially the later charts of atlases on $n D$ differential manifolds-, Riemann then by hypothesis set off with a quite arbitrary RiemannFinsler "geometrical structure" or line element $d s$ and, to get more explicit and to go for most possible simplicity in his exposition, further specified his hypothesis to $a$ positive definite metric tensor $g$ given in classical notation by $g=g_{h k} d x^{h} d x^{k}$, whereas Helmholtz straightforwardly came up with this same latter geometrical structure, i.e. at once set off with a squared line element given by a generalised theorem of Pythagoras as a general quadratic homogeneous polynomial in infinitesimal changes of the co-ordinates as variables, because he found this to be the only factual possibility to allow for measurements of distances invariant under congruences. The intrinsic geometry of surfaces $M^{2}$ in $E^{3}$ thus having been the inspiration for the creation of the more general Riemannian geometry on $2 D$ differential manifolds (cfr. e.g. Vincent Borrelli e.a.'s "tore plat en $3 D$ "), i.e. of $2 D$ Riemannian spaces, may show how human's geometrical experiences in "their space" actually have influenced their definition of abstract metrical Riemannian spaces $\left(M^{n}, g\right)$ of arbitrary dimension $n$.

The successful demonstration of the existence of non-Euclidean geometries, which had been understood right away by the few mathematicians who in private talked and corresponded about the controversial parallel postulate amongst each other, at last became clear for all after Beltrami's publication in 1868 of the intimate link between the validity of the classical non-Euclidean hyperbolic geometry on the one hand and the validity of Euclidean geometry itself on the other. And this brought along the profound and quite revolutionary re-evaluation of the whole field of mathematical logic on which, till at present, we can pretty well base actual confidence when doing our mathematical doings.

Following Minkowski's 1908 Köln lecture on "Raum und Zeit", Riemannian geometry was put in the indefinite setting that became the language in which Einstein in 1916 could formulate his general theory of relativity, (which may explain the above 
notation $g$ for $d s^{2}$ ); actually, Einstein's field equations basically define mass and energy in terms of the Ricci curvatures of "our" physical spacetime. The opening lines of this lecture will remain remembered forever: "M. H.! Die Anschauungen über Raum und Zeit die ich Ihnen entwickeln möchte, sind auf experimentell-physikalischen Boden erwachsen. Darin lieght ihre Stärke. Ihre Tendenz ist eine Radikale. Von Stund an sollen Raum für sich und Zeit für sich völlig zu Schatten herabsinken, und nur noch eine Art Union der beiden soll Selbständigkeit bewahren". And, our kind's perceptions of the space dimensions, or, say, of the space co-ordinates $x, y$ and $z$, and of the time dimension, or, say, of the time co-ordinate $t$, naturally being of different natures, in Minkowski's determination of a Pythagorean measure of the distances in the $(x, y, z ; t)$ spacetime according to the Weltpostulat, basically the three letter combination ict showed up for the very first time, and, as he put it: "Man kann danach das Wesen dieses Postulates mathematisch sehr prägnant in die magische Formel kleiden: $300000 \mathrm{~km}=$ i sek". In local co-ordinates $(x, y, z ; t)$ thus originated the Minkowski metric $d s^{2}=d x^{2}+d y^{2}+d z^{2}-d t^{2}$ of index 1 as first indefinite revision of the classical theorem of Pythagoras. And accordingly could begin the development of pseudo Riemannian geometry along the lines of proper, definite Riemannian geometry. The proper Euclidean, respectively the proper Riemannian spaces, in some sense conversely, then are the pseudo Euclidean, respectively the pseudo Riemannian spaces of index 0. In his thesis with Carathéodory, Finsler initiated the systematic study of "his" geometry, which recently became more widely accessible thanks to the research of Chern and co-workers.

And, mostly from Helmholtz's thoughts about a role to be played in Riemannian spaces $\left(M^{n}, g\right)$ by congruences, (like in the sense of classical Euclidean spaces $E^{n}$ ), as a necessity to actually allow for measurements of distances "in a normal way", resulted the characterisation by Riemann, Helmholtz, Lie, Klein and Tits of the utmost possible symmetrical Riemannian spaces, i.e. of the spaces which look the same at all their points and which at every point look the same in all directions, or, still, of the perfectly homogeneous and isotropic spaces, as the spaces $\left(M^{n}, g\right)$ of constant Riemannian or sectional curvature $K$, say $K=c$ (for possibly c $=\mathrm{o}, c>$ o and $c<0$ ), these "real space forms" then being denoted by $M^{n}(c)$. Or, still, the spaces $M^{n}(c)$ are the Riemannian spaces which satisfy the axiom of free mobility, i.e. for which "the measurements of all beings living in such spaces do not change however whereabouts these beings may move to in these spaces". According to what is known as the theorem of Beltrami, the real space forms $M^{n}(c)$ are the Riemannian manifolds $\left(M^{n}, g\right)$ which are projectively equivalent to the locally Euclidean spaces. And these spaces $M^{n}(c)$ all are very nice geometrical models indeed for "our real outside worlds" since, as discussed in particular by Klein, in view of the threshold of our sense perceptions and the fact that our space perception is adapted to a limited part of space only, our space perception can be described as closely as desired by real space forms of zero, positive or negative curvatures $c$ alike. The above studies are highlights among the efforts to well answer the question of how to objectively 
describe in a reasonable way "the theatre in which we have the impression to be on stage", which, like in antiquity, again took up a central position in scientific research since the second half of the 19th century. In the words of Chern: "While algebra and analysis provide the foundations of mathematics, geometry is at the core".

In the same way as Euclidean geometry in dimension 2 essentially derives from the circle as ground figure by which distances may be determined in an isotropic way when considered from a human point of view, the Lamé curves (which were called supercircles by Piet Hein) are at the basis of the simplest definite Minkowski-Finsler geometries in which 4 -fold anisotropies occur when considered from a human point of view, and analogous observations can be made in dimensions 3 and more. In this respect, the so-called Gielis curves for dimension 2 and the Gielis (hyper)surfaces for dimension 3 (and more) turned out to be ground figures for describing most natural $\mathrm{s}$-fold anisotropies (for $s=0,1,2,3,4,5, \ldots$, or, for that matter, for any $s \in R$ ). And, closely related herewith, by application of the corresponding Gielis transformations to the "most natural" curves and surfaces of Euclidean geometry (e.g., for dimension 2: the circles amongst the closed curves and the logarithmic spirals amongst the nonclosed curves), do result many of the forms that we do observe in nature -in biology, cristallography, physics, chemistry, etc.- Hereby, a motivation of "Euclidean most natural" fitting in as special case of Bang-Yen Chen's recent theory of "submanifolds of constant ratio", being that in the evolution of these shapes of any dimension $n$ starting off out of a pole, say the origin $O$ of a Euclidean ambient space of any dimension $n+m$, the length of the gradient of the polar distance always remains the same, which for curves in a Euclidean plane amounts to D'Arcy Thompson's basic principle of growth in biology.

Roughly speaking, the main algebraic-analytic formalisms employed in differential geometry are threefold. First, one has the Ricci calculus, which essentially concerns tensor calculus in local co-ordinates; it was developed a.o. by Ricci, Levi-Civita, Schouten and Struik. Next, one has the Cartan calculus or $\omega$-calculus, (Cartan often denoted the connection differential forms and the curvature differential forms by omega's), which basically is the method of "the moving frames". It goes back to Euler's view on the meaning of the curvature of Euclidean planar curves and then was further developed by Pagani, Frenet, Serret, Ribeaucour and Darboux for curves and surfaces in Euclidean $3 D$ space. It was intrinsically extended to full generality by É. Cartan, following Lamé's studies using curvilinear co-ordinates for the 3-dimensional Euclidean spaces themselves and Demoulin's likewise studies of projective spaces. And, only recently, it was extended extrinsically to full generality too, of which the main step, the determination of the geometrically most adapted principal orthonormal frame fields on submanifolds of arbitrary dimensions and co-dimensions, was made by Kostadin Trenčevski; the best choices of moving frames before had been done almost one and a half century ago by Camille Jordan for dimension 1 and arbitrary co-dimensions, (i.e. for curves in general), and by Kronecker and by Jordan for submanifolds of arbitrary dimensions and, respectively, of co-dimension 1 and of 
arbitrary co-dimensions, in the latter case however, as indicated above, till only recently having been restricted to the tangential parts of such frames only. Finally, one has the nabla-calculus or the co-ordinate invariant calculus, which was thought of at Chicago by Koszul and fully developed there by Katsumi Nomizu in the 19fifties. The importance of well designed formalisms may not be underestimated: for a geometer whose intuition is perfectly in tune with one of such formalisms, its playful use in doing research may sometimes be like interactive, in that it may sometimes like guide and inspire this geometer further than he would have imagined to be able to get in the first place or indicate new directions to be explored completely beyond his scope when starting to work. And though every geometer is at ease with each of the methodologies of his field, most geometers are inclined to favour one of the three when studying some problems which are neutral with respect to methodology; for instance, amongst the students of Cartan who became themselves leading geometers in their own right with remaining contributions to intrinsic and extrinsic geometry, Kentaro Yano and Radu Rosca were champions in tensor calculus and in omega calculus, respectively.

Generalising the 1917 (pseudo) parallel transport of Levi-Civita (and Schouten, 1918) of tangent vectors along curves in (pseudo) Riemannian manifolds, in some sense as farthest thinkable reasonable extension of Euclidean geometry up till now, after some two millennia of reflecting on the parallel postulate, one further came to the study of the geometry of general connections in fibre bundles. But, according to Chern and in any case in our present times, Riemannian geometry is the central topic in geometry. And, by the 1956 isometrical embedding theorem of Nash, every abstract $n$-dimensional (definite) Riemannian manifold can, in many ways, be considered as a submanifold in Euclidean spaces of sufficiently high dimensions $n+m$, and a Lorentzian version and the general indefinite Riemannian manifold version of this fundamental result concerning isometrical embeddings in appropriate pseudo Euclidean spaces of sufficiently high dimensions were later given by Friedman and by Clarke and Greene. Thus, (pseudo) Riemannian geometry conceptually essentially is equivalent with the intrinsic geometry of the (pseudo) Riemannian submanifolds of arbitrary dimensions $n$ and co-dimensions $m$ in (pseudo) Euclidean spaces, and, as such, is part of the geometry of submanifolds of (pseudo) Euclidean spaces which itself can be seen for arbitrary dimensions and co-dimensions in our natural imagination to correspond to the abstraction of our basic visual and motoric sense-experiences of "the real curves and the real surfaces that we do encounter in our real worlds". In this respect, from Jacob Bronowski's "The Origins of Knowledge and Imagination" come the following complementary quotes: "The place of sight in human evolution is cardinal" and "The world of science is wholly dominated by the sense of sight", and in the same author's chapter "On the music of the spheres" in his book "The Ascent of Man" one finds a so beautiful discussion on the Euclidean theorem of Pythagoras as wonderful connection between the two. In this light, the appreciation of the geometry of submanifolds as the geometry of our human kind may be seen in not too misty a way 
after all. Further along these lines, one might consider the goals of Hugo Steinhaus' "Kaleidoskop der Mathematik": to show that (1) "Der Gegenstand der Mathematik ist die Wirklichkeit" and that (2) "Die Mathematik ist universell". And, in his "La valeur de la science", Henri Poincaré like rounded off observations of the sorts just made by the following statements: "Ce que nous appelous la réalité objective, c'est, en derniè̀e analyse, ce qui est commun à plusieres êtres pensants, et pourrait être commun à tous; cette partie commune ne peut être que l'harmonie exprimée par les lois mathématiques" and "La pensée n'est qu'un éclair au milieu d'une longue nuit. Mais c'est cet éclair qui est tout".

In the geometry of general submanifolds $M^{n}$ in Euclidean spaces $E^{n+m}$, or in general (pseudo) Riemannian ambient spaces $\tilde{M}^{n+m}$ for that matter, the knowledge of fundamental links between the intrinsic and the extrinsic geometries of submanifolds is crucial. In the 19nineties, Bang-Yen Chen initiated significant advances in this respect, to begin with in terms of his new scalar valued Riemannian curvature invariants, by deriving general optimal inequalities between various intrinsic and extrinsic characteristics of submanifolds (cfr. B.-Y. Chen, "Pseudo-Riemannian Geometry, $\delta$-invariants and Applications", World Scientific Publ. Co., Singapore, 2011). The corresponding Chen and Wintgen ideal submanifolds, i.e. the shapes which are assumed by given abstract Riemannian manifolds when realising them in Euclidean spaces as submanifolds for which the extrinsic tensions (which for the Chen ideal submanifolds involve the squared mean curvature and for the Wintgen ideal submanifolds moreover involve the curvature of the normal bundle) are as small as possible, turn out to manifest intrinsic symmetry properties of a special basic type studied since the 19eighties, in particular by Ryszard Deszcz. When these properties concern the Riemann curvature tensor, the manifolds involved are called Deszcz symmetric spaces, and the following brief introduction to such spaces, leading up to quasi-umbilical hypersurfaces in space forms as visualisations of "our theatre" in the above sense, will end the first part of this little hi-story of geometry.

The fundamental lemma of Riemannian geometry states that there exists a unique Riemannian connection $\nabla$, i.e. a derivation on any Riemannian manifold $\left(M^{n}, g\right)$ of vector fields with respect to vector fields following the rules of linearity and the product rule of Leibniz and which is compatible with the differential structure on $M^{n}$, (in that the commutator of this connection is identical with the Lie bracket of vector fields), and which, as well is compatible with the geometrical structure $g$ on $\left(M^{n}, g\right)$, (in that $\nabla g=0$; -from deriving vector fields by $\nabla$ one can normally get to deriving arbitrary tensor fields by $\nabla$-). This Riemannian connection $\nabla$ is given by the standard formula of Koszul and the corresponding expressions for the Riemann-Christoffel, for the Ricci and for the Weyl conformal curvature tensors $R, S$ and $C$ respectively, etc., were systematically developed by Nomizu in his thesis with Chern. The Riemannian or sectional curvatures $K(p, \pi)$ were known to be scalar valued isometric invariants of $\left(M^{n}, g\right)$, determined at any point $p$ and for any $2 D$ tangent plane section $\pi$ at $p$, right away since their very introduction by Riemann, but their name "curvature" 
merely derived from the analogy of their calculation with the intrinsic formula for the Gauss curvature $K$ of surfaces $M^{2}$ in $E^{3}$, now applied for the Gauss curvature at $p$ of the $2 D$ surface $G^{2}$ formed around $p$ by the geodesics of $\left(M^{n}, g\right)$ which at $p$ are tangent to $\pi$. Of course, as regards further appreciations of curvatures $K$, one could also base e.g. on the formulas of Bertrand-Puisseux and of Diguet referring to the perimeters or the areas of geodesic circles or discs on $\left(M^{n}, g\right)$ in comparison with the perimeters and areas of Euclidean circles and discs of the same radii. But striving for better truly geometrical insights in the curvature tensor $R$ or equivalently in the sectional curvatures $K$, as already mentioned before, around the same time and independently, Levi-Civita and Schouten introduced the notion of parallel (or pseudo parallel) transport of vectors along curves in $\left(M^{n}, g\right)$, -which is equivalent with the notion of Riemannian connection- to obtain their geometrical interpretations of $R$ and $K$ in terms of the lengths of the sides and the areas of parallelogramoids and of holonomy of vectors or of directions, respectively.

Pretty oversimplifying for instance Weyl's view on this matter in his "Symmetry" book, it could be stated that "symmetry means that something keeps the same measure after something has been done with it". As such essentially, "the something that we want to do with things", i.e. the transformations or operations that we want to consider in the following discussions on symmetry of Riemannian spaces are the parallel transports around infinitesimal co-ordinate parallelograms. These likely are the most natural transformations to perform on Riemannian manifolds $\left(M^{n}, g\right)$ when showing proper respect for both the differential and the metrical structures of these spaces; (cfr. the chapter "On Natural Symmetries" with Ryszard Deszcz and Stefan Haesen in the Ed. Acad. Romania's "Topics in Differential Geometry", 2008, Eds. A. and I. Mihai and R. Miron, and cfr. the article "Natural Intrinsic Geometric Symmetries" with Stefan Haesen in the 2009 SIGMA special volume on "Élie Cartan and Differential Geometry", where these matters are dealt with a bit more delicately as compared to the sometimes rather rough formulations that will come hereafter).

After thus having decided on the type of transformations for which we will consider corresponding symmetries, next we should choose "what kind of things" actually to transform. And to begin with, as Schouten was first to think of, long ago, the very simplest "beings" to parallely move around, of course, are vectors. As is well known, certainly since the general use of the $\nabla$ formalism in most current introductions to Riemannian geometry, it in fact became the definition self of the Riemann-Christoffel curvature tensor: $R$ measures the difference between any vector $\vec{v}$ at any point $p$ of a Riemannian space $\left(M^{n}, g\right)$ and the corresponding vector $\vec{v}_{\bar{\pi}}^{*}$ which results from parallely moving $\vec{v}$ completely around any infinitesimal co-ordinate parallelogram $\mathcal{P}$ which is "cornered" at $p$ and whose sides at $p$ are tangent to any non co-linear vectors $\vec{x}$ and $\vec{y}$ at $p$, (and before we have already denoted $\bar{\pi}$ for the tangent 2-plane $\bar{\pi}=\vec{x} \wedge \vec{y}$ ); or, still, in a terminology of Élie Cartan: $R$ constitutes the holonomy (from "holos" and "nomos") for vectors or equivalently for tangent directions on Riemannian manifolds $\left(M^{n}, g\right)$ under their parallel transports all around infinitesimal 
co-ordinate parallelograms. And, by a theorem of Cartan, the information contained in $R$ is the same as the information given by the sectional curvatures $K(p, \pi)$ for all points $p$ and for all $2 D$ planes $\pi$ tangent to $M^{n}$ at $p$. So, in conclusion of the above: the Riemannian manifolds $\left(M^{n}, g\right)$ which satisfy the symmetry property that all their directions at all their points remain invariant under all such parallel transports are the locally flat or locally Euclidean spaces, i.e. the Riemannian spaces for which $R=0$ or, equivalently, for which $K=\mathrm{o}$. In passing, it could be worthwhile to point our here that, in the mean time, via the theorem of Nash, various aspects of interest concerning abstract Riemannian manifolds $\left(M^{n}, g\right)$ can well be interpreted pretty "concretely" when actually viewing these manifolds as submanifolds in ambient Euclidean spaces $E^{n+m}$. As such, in particular, any sectional curvature $K(p, \pi)$ of $\left(M^{n}, g\right)$ can be seen as the Gauss curvature at $p$ of the $2 D$ surface $\Sigma^{2}$ in $E^{2+m}$ which is the $2 D$ normal section at $p$ of the submanifold $\left(M^{n}, g\right)$ of $E^{n+m}$ by the $(2+m) D$ normal space to this submanifold spanned by the normal space $T_{p}^{\perp} M^{n}$ as such to the submanifold $M^{n}$ in $E^{n+m}$ at $p$ together with the $2 D$ tangent plane $\pi$ to this submanifold at $p$. And the Gauss curvature at $p$ of such surfaces $\Sigma^{2}$ in $E^{2+m}$ may further be "reduced" to Gauss curvatures at $p$ of surfaces $\Sigma_{\xi}^{2}$ in $3 \mathrm{D}$ Euclidean spaces $E^{3}$, these latter surfaces $\Sigma_{\xi}^{2}$ resulting by projecting surfaces $\Sigma^{2}$ in $E^{2+m}$ onto the subspaces $E^{3}$ of $E^{2+m}$ which are spanned at $p$ by $\pi$ together with any normal $\xi$ to $M^{n}$ in $E^{n+m}$ at $p$. And, finally, in some sense closing up a sort of "curvature-circle" which in a way started with Newton's determination of the curvature of Euclidean planar curves, via Euler's normal ( $1 D)$ sections of surfaces $M^{2}$ in $E^{3}$ and thus via the principal curvatures $k_{1}$ and $k_{2}$ of such surfaces a.o. came to the intrinsic Gauss curvature $K=k_{1} \cdot k_{2}$, the extrinsic mean curvature of Germain $H=\left(k_{1}+k_{2}\right) / 2$ which relates to the surface tension that results from the surface's shape in $E^{3}$ and the extrinsic curvature of Casorati $C=\left(k_{1}^{2}+k_{2}^{2}\right) / 2$ which, in best accordance with our visual intuition and common sense, relates to the form proper of surfaces $M^{2}$ in $E^{3}$, and then via Gauss' theorema egregium formula, with Riemann, led to the notion of the sectional curvatures $K$ of his manifolds $\left(M^{n}, g\right)$, which now, in turn, reducible back to Gauss curvatures of surfaces $\Sigma_{\xi}^{2}$ in $E^{3}$, can be determined by curvatures of Euclidean planar curves, or, restating this last step more precisely: the Gauss curvature of any surface $M^{2}$ in $E^{3}$ at any of its points is, up to sign, the squared curvature of a Euclidean planar normal section (which section can be easily determined using Euler's formula).

Now, let it be recalled that the locally Euclidean spaces are characterised among all Riemannian manifolds by the symmetry property of the invariance of their directions under the parallel transports around their infinitesimal co-ordinate parallelograms. And, as also was mentioned before: the projective class of the locally Euclidean Riemannian manifolds consists of the Riemannian spaces of constant curvature $K=c$, or, still, of the real space forms $M^{n}(c)$, (flat or parabolic for $\mathrm{c}=\mathrm{o}$, elliptic for $c>0$ and hyperbolic for $c<0$ ). In other words, by application of projective transformations to the locally flat spaces one obtains real space forms and the class of real space forms 
is closed under projective transformations. At this stage it might be not unappropriate to reflect a while on the geometrical meaning of projective transformations also in relation with the fundamental naturalness in our kind's visual and motoric abilities to metrically look at the same things from different points of view. Moreover, it could be remarked here and now that, in view of the projective models of the Euclidean and of the elliptic and hyperbolic non-Euclidean geometries which were established prior to the above recalled theorem of Beltrami, Cayley made the following observation: "Metric geometry is a part of descriptive geometry, and descriptive geometry is all geometry", herewith in his way neatly illustrating the psychologically based interwoveness of "our" visual experiences and of "our" geometries. In any case, the Euclidean and the non-Euclidean real space forms $M^{n}(c)$, -by Schur's lemma, for dimensions $n \geq 3$, the Riemannian manifolds $\left(M^{n}, g\right)$ for which the main Riemannian invariant, the Riemannian or sectional curvature function, $K(p, \pi)$ is isotropic in the sense as to be the same for all $2 D$ planes $\pi$ at any of its points-, constitute the projective class of the Riemannian manifolds for which likely their simplest geometrical objects, namely their tangent directions, remain invariant under likely their most natural transformations, namely the parallel transports around infinitesimal co-ordinate parallelograms. As stated before, it are the spaces $\left(M^{n}, g\right)$ satisfying the axiom of free mobility, or, still, the spaces $\left(M^{n}, g\right)$ whose group of isometries has the maximal possible dimension $d=n(n+1) / 2$, and, speaking about symmetry in its plainest meaning of our common sense: these spaces look the same at all of their points and at every point these spaces look the same in all directions, being thus both in the mathematical sense and in the common sense likely the most perfect spaces that we could imagine, when allowing to leave the boundaries of Euclidean geometry per se, and which do correspond to our most immediate and unsophisticated description of "the world of our human sight", and, as touched upon before, these kinds of speculations could be carried through too for the indefinite case as well. The geometry of "our" nature however, in several factual situations, sometimes also drastically opposes to the formerly described ultimate mathematical perfection. For instance, "thinking large", and, regarding the spacetimes of the general theory of relativity, thinking for instance of the FriedmannLemaitre cosmological models of the 19twenties, isotropy as such -all $1 D$ directions being the same- obviously cannot be expected to occur: in particular such isotropy here implying that it would be impossible to distinguish between the "wheres" and the "whens" of any event. And, for instance, "thinking small", the homochiralities in biochemistry, going back to a discovery of Pasteur in 1848 and probably best known from DNA molecules with torsions exclusively being of only one sign, naturally may appear to us as in conflict with the mirror or bilateral symmetries which are so significantly present in the real space forms indeed. Thus it came about to look for, in some sense, "the second best possible symmetric spaces", or, still, "the anisotropic spaces which are most symmetrical", properly determined from within geometry itself and such that the main symmetries of these spaces would a.o. better tolerate the kinds of directional or orientational preferences that just have been mentioned. In the above 
contexts, a rather reasonable way to do so could well be the following. Here is the opening paragraph of Robert Osserman's "Curvature in the Eighties": "The notion of curvature is one of the central concepts of differential geometry; one could argue that it is the central one, distinguishing the geometrical core of the subject from those aspects that are analytic, algebraic, or topological. In the words of Marcel Berger, curvature is the number one Riemannian invariant and the most natural. Gauss and then Riemann saw it instantly"; and for a panoramic view of Riemannian geometry, see Marcel Berger's book "A Panoramic View of Riemannian geometry", Springer, Berlin, 2003). And, also directly from these words alone, the real space forms $M^{n}(c)$ present themselves as the most perfect Riemannian spaces, their most important Riemannian invariant $K(p, \pi)$ being the same at all points $p$ and in all $2 D$ directions $\pi$. So, next, we take a keen interest in the Riemannian spaces $\left(M^{n}, g\right)$ for which the sectional curvatures $K(p, \pi)$ are invariant under the parallel transports around all infinitesimal co-ordinate parallelograms $\mathcal{P}$ cornered at $p$ and tangent to all planes $\bar{\pi}$. As noted with Stefan Haesen, these Riemannian spaces turn out to be the so-called semi symmetric spaces, i.e. the spaces for which $R \cdot R=0$ whereby the first $R$ stands for the curvature operator and the second $R$ stands for the $(0,4)$ Riemann-Christoffel curvature tensor (thus meaning that for all vector fields $X$ and $Y: R(X, Y) \cdot R=0$, $R(X, Y)=\nabla_{X} \nabla_{Y}-\nabla_{Y} \nabla X-\nabla_{[X, Y]}$ hereby acting as a derivation on $R$ ). The class of the semi symmetric spaces evidently encloses the class of Cartan's locally symmetric spaces, which are defined as the Riemannian manifolds for which $\nabla R=0$, and which geometrically correspond to the geodesic symmetries around all points of such spaces being local isometries; Zoltan Szabó gave a full classification of the semi symmetric spaces in the 19eighties while the locally symmetric spaces had been fully classified long before by Élie Cartan. Of course, the semi symmetric spaces contain the real space forms as their most trivial examples. As shown by Levy in 1926, the Cartan or locally symmetric Riemannian spaces are characterised by the fact that their sectional curvatures are invariant under the parallel transport along all infinitesimal line segments; in a loose way, this visualises into these curvatures remaining the same under the reflections in all infinitesimally nearby mirrors. And, in this way looking at things, for semi symmetry the geometric transformations, the invariance of the Riemannian curvatures under which is the defining characteristic, are the compositions of four parallel transports along such line segments, namely along the consecutive sides of the co-ordinate parallelograms $\mathcal{P}$.

And now, in a manner of speech in a sense as used before, we finally consider the class of the so-called pseudo symmetric or Deszcz symmetric spaces, which spaces are seen when looking at the semi symmetric or Szabó symmetric spaces from different points of view. When applying a projective transformation to a semi symmetric space, in general, the semi symmetry condition $R \cdot R=0$ is no longer satisfied, but after such transformation the resulting Riemannian manifold's $(0,6)$ curvature tensors $R \cdot R$ and $\wedge_{g} \cdot R$, whereby $\wedge_{g}$ stands for the natural metrical endomorphism, are proportional, which is the tensorial definition of pseudo symmetry, and the class 
of all pseudo symmetric spaces itself is closed under projective transformations. The step from the semi symmetric to the pseudo symmetric spaces basically is completely analogous with the step from the locally Euclidean spaces $(K=0)$ to the real space forms ( $\mathrm{K}$ is constant; $\mathrm{c}=\mathrm{O}$ or $c>0$ or $c<\mathrm{o}$ ). Just as the Riemann-Christoffel $(0,4)$ curvature tensor $R$ is fully determined by the Riemannian or sectional curvatures $K(p, \pi)$, the $(0,6)$ curvature tensor $R \cdot R$ is fully determined by the so-called Deszcz or double sectional curvatures $L(p, \pi, \bar{\pi})$; (these latter were introduced with Stefan Haesen in the paper "Properties of a scalar curvature invariant depending on two planes", manuscripta math. 122, 2007). Unlike for the sectional curvatures $K(p, \pi)$ there does not hold a lemma of Schur for the double sectional curvatures $L(p, \pi, \bar{\pi})$; but apart from this, just like real space forms -at least for $n \geq 3$ - are characterised by the fact that their curvatures $K(p, \pi)$ do not depend on the planes $\pi$, (say by the fact that their sectional curvatures are isotropic, i.e. do not depend on the choice of $2 D$ directions $\pi$ ), the Deszcz symmetric spaces are characterised by the fact that their curvatures $L(p, \pi, \bar{\pi})$ do not depend on the planes $\pi$ and $\bar{\pi}$, (i.e. by the fact that their double sectional curvatures are isotropic, namely do not depend on the choice of the $2 D$ directions $\pi$ and $\bar{\pi}$ ). And $L=o$ characterises the Szabó or semi symmetric spaces. In the same notations as used before, the invariance of the curvatures $K(p, \pi)$ under parallel transports around infinitesimal co-ordinate parallelograms $\mathcal{P}$ cornered at $p$ and tangent there to a plane $\bar{\pi}=\vec{x} \wedge \vec{y}$ means the following: when the plane $\pi$ is spanned by two vectors $\vec{v}$ and $\vec{w}$ at $p, \pi=\vec{v} \wedge \vec{w}$, after the parallel transports of $\vec{v}$ and $\vec{w}$ around $\mathcal{P}$, although in general the plane $\pi_{\vec{\pi}}^{*}$ spanned by the then obtained vectors $\vec{v}_{\bar{\pi}}^{*}$ and $\vec{w}_{\bar{\pi}}^{*}$ at $p, \pi_{\bar{\pi}}^{*}=\vec{v}_{\bar{\pi}}^{*} \wedge \vec{w}_{\bar{\pi}}^{*}$, is different from the original plane $\pi$, the sectional curvatures of both these planes at $p$ are the same: $K\left(p, \pi_{\bar{\pi}}^{*}\right)=K(p, \pi)$. And, in the same sense that the sectional curvatures measure the change of directions under the parallel transports around parallelograms $\mathcal{P}$, the double sectional curvatures measure the change of sectional curvatures under such parallel transports.

In the standard way, in particular what was done in the last few paragraphs, may be readily repeated also for the indefinite case, i.e. the above semi symmetry and pseudo symmetry conditions can be considered equally well in the pseudo Riemannian setting. And, as such, for instance in the case of $4 D$ Lorentzian geometry, most of the physically relevant spacetimes which have been studied so far turn out to be Deszcz symmetric spaces indeed like i.p. the Friedman-Lemaitre spacetimes. Besides, and hereby again focusing in the following on proper Riemannian geometry, next come some further comments about the "mild" anisotropies as manifested by the nontrivial pseudo symmetric spaces, i.e. by the pseudo symmetric spaces which do not have constant Riemannian curvatures $K$, (these latter spaces being fully isotropic, all their tangent directions enjoying a same status, all enjoying the same geometric qualities). A $3 D$ Riemannian manifold $\left(M^{3}, g\right)$ is pseudo symmetric if and only if it is quasi Einstein, i.e. if and only if its Ricci tensor $S$ has an eigenvalue, or, still, a Ricci principal curvature, of multiplicity $\geq 2$. In particular, when $\left(M^{3}, g\right)$ is Einstein, i.e. when its Ricci tensor $S$ is proportional to the metric tensor $g$, or, still, when all tangent 
directions of $\left(M^{3}, g\right)$ have the same Ricci curvatures, by a theorem of Schouten and Struik, $\left(M^{3}, g\right)$ then is a real space form; (hereby the property to be Einstein and the isotropy of $1 D$ directions amounting to the same, in this very special situation). But on the non-trivial pseudo symmetric spaces of dimension 3, the properly quasi Einstein spaces, the tangent spaces split up in a $2 D$ plane and its orthogonal $1 D$ line which are then the two eigenspaces of $S$ belonging to the then two distinct Ricci principal curvatures of multiplicities 2 and 1, respectively. In particular, as such are also the five non-trivial $3 D$ Thurston spaces, and it moreover could be noted here that their Deszcz curvatures are constant; (more precisely $\mathrm{L}=0,+1$ or -1 ). On a randomly choosen $3 D$ Riemannian manifold on the other hand, the Ricci tensor has 3 mutually distinct principal curvatures with 3 mutually orthogonal principal $1 D$ directions, thus showing in a sense a "wild" anisotropy, as such spaces geometrically do behave quite differently in almost all directions.

In the present paragraph we will only consider Riemannian spaces $\left(M^{n}, g\right)$ of $d i$ mensions $n>3$ which are conformally flat or, using some earlier formulation, spaces which in their smallest parts are similar to Euclidean spaces, or, still, to the spaces $\left(M^{n}, g\right)$ of dimension $n>3$ with vanishing Weyl conformal curvature tensor $C$. Such spaces are Deszcz symmetric if and only if they are "partially" Einstein, i.e. if and only if their Ricci tensor $S$ has at most two distinct principal values. In the particular case of Einstein spaces proper, as is well known, then again we are dealing with the real space forms and there is full $1 D$ isotropy. In the non-trivial case however, the tangent spaces of the Deszcz symmetric conformally flat manifolds split up in two orthogonally complementary subspaces $S_{1}$ and $S_{2}$, namely the eigenspaces belonging to the two distinct Ricci curvatures, say $\rho_{1}$ and $\rho_{2}$, with dimensions $n_{1}$ and $n_{2}$, respectively, the multiplicities of $\rho_{1}$ and $\rho_{2}$; and in directions of $S_{1}$ as compared to in directions of $S_{2}$ such spaces look essentially different, but all directions of $S_{1}$ are equivalent among each other and likewise all directions of $S_{2}$ are equivalent among each other, which expresses the meaning of the mild anisotropy that was alluded to before. After the real space forms, the most special conformally flat Deszcz symmetric spaces likely are those which are properly quasi Einstein, i.e. those for which, say, $n_{1}=1$ and $n_{2}=n-1$, i.e. for which there is a unique privileged direction $S_{1}$, the principal Ricci direction corresponding to the Ricci curvature $\rho_{1}$ of multiplicity 1 , and all directions perpendicular to $S_{1}$ are mutually the same, or, still, all directions of the $(n-1) D$ eigenspace $S_{2}$ of the Ricci tensor do share the same properties among each other, but these properties are different from those that the direction $S_{1}$ in some sense enjoys on its own. Now, as was basically shown already in the early 19seventies by B.-Y. Chen, K. Yano and C.-S. Houh, these special conformally flat Deszcz symmetric spaces are what -by a pretty well choosen name for that matter- at present are called the spaces with quasi constant sectional curvatures, and already then, they moreover identified these Riemannian spaces $\left(M^{n}, g\right)$ with the quasi umbilical hypersurfaces $M^{n}$ in the real space forms $M^{n}(c)$, or, still, with the loci of spheres in the real space forms, (cfr. Bang-Yen Chen's chapter 3 on "Riemannian Submanifolds" in "Handbook of 
Differential Geometry, Volume 1", Elsevier, A'dam, 2000, Eds. Franki Dillen e.a.). In particular, in Euclidean ambient spaces $E^{n+1}$, the special pseudo symmetric spaces $\left(M^{n}, g\right)$ concerned can thus be visualised as the envelopes of 1-parameter families of spheres, and, when $\lambda$ and $\mu$ denote the principal curvatures of these proper quasi umbilical hypersurfaces $M^{n}$ in $E^{n+1}$, then one can also readily visualise their Deszcz sectional curvature too, namely as being given by $L=\lambda \mu$.

The Dutch word for "geometry" is "meetkunde", which may actually much better than the term geometry itself indicate what geometry is really all about. "To measure the earth" was and remains a fascinating endeavor, and, for instance, Mercator's projection for ever will remain a great example of meetkunde, both from the pure and from the applied points of view. But meetkunde, literally, stands for "the art to achieve measure, balance, what is just (meaning here correct as well as precise)", or, still, meetkunde stands for "the art to see things right or to see things as they really are" (which should be interpreted in the context of our understanding of human sensations and perceptions), and the Dutch "meten" means "to measure", i.e. to take the measure, i.e. to take, in Dutch, "de maat", and "maât" was the name of an Egyptian goddess of justice. Maybe in other languages such better terms are in use as well; in any case, Kepler tried in vain to introduce the term "Mass-Kunst" also in German, and, as far as I know, "mértan" means meetkunde in Hungarian.

(to be continued)

KU LEUVEN,

SECTiOn OF GeOMETRY,

BELGIUM

E-mail address: leopold.verstraelen@wis.kuleuven.be 